\title{
Association between season of vaccination and antibody levels against infectious diseases
}

\author{
T. C. Abreu ${ }^{1}$ (D) H. Boshuizen ${ }^{2}$, L. Mollema ${ }^{1}$, G. A. M. Berbers ${ }^{1}$ and \\ H. Korthals Altes ${ }^{1}$
}

\section{Original Paper}

Cite this article: Abreu TC, Boshuizen $\mathrm{H}$, Mollema L, Berbers GAM, Korthals Altes $\mathrm{H}$ (2020). Association between season of vaccination and antibody levels against infectious diseases. Epidemiology and Infection 148, e276, 1-10. https://doi.org/10.1017/ S0950268820002691

Received: 7 April 2020

Revised: 2 October 2020

Accepted: 15 October 2020

\section{Key words:}

Childhood vaccination schedule; seasonality; serology; vaccine

Author for correspondence:

T. C. Abreu,

E-mail: t.c.abreu@amsterdamumc.nl (c) The Author(s), 2020. Published by Cambridge University Press. This is an Open Access article, distributed under the terms of the Creative Commons Attribution licence (http://creativecommons.org/licenses/by/4.0/), which permits unrestricted re-use, distribution, and reproduction in any medium, provided the original work is properly cited.

\section{CAMBRIDGE} UNIVERSITY PRESS
${ }^{1}$ Centre for Infectious Disease Control, National Institute for Public Health and the Environment (RIVM), Bilthoven, the Netherlands and ${ }^{2}$ Centre for Nutrition, Prevention and Health Services, National Institute for Public Health and the Environment (RIVM), Bilthoven, the Netherlands

\begin{abstract}
Vaccination has reduced the disease burden of vaccine-preventable diseases. However, the extent to which seasonal cycles of immunity could influence vaccine-induced immunity is not well understood. A national cross-sectional serosurveillance study performed in the Netherlands (Pienter-2) yielded data to investigate whether season of vaccination was associated with antibody responses induced by DT-IPV (diphtheria, tetanus and poliomyelitis), MMR (measles, mumps and rubella) and meningococcus $\mathrm{C}$ (MenC) vaccines in children. In total, 434 children met the inclusion criteria to study DT-IPV immunity, 811 for MMR and 311 for MenC. Differences in $\log$ (antibody levels) by season of vaccination were investigated with linear multivariable regression analyses. Seroconversion rates varied according to season of vaccination for rubella ( $90 \%$ of autumn-vaccinated children vs. $99 \%$ of winter-vaccinated had concentrations above cut-off levels). Summer-vaccinated boys showed a slower decline of tetanus antibodies ( $6 \%$ per month), in comparison with winter-vaccinated boys. In conclusion, season of vaccination showed little association with immunological protection. However, a number of associations were seen with a $P$-value of about 0.03; and adding data from a just-completed nationwide serological study might add more power to the current study. Further immunological and longitudinal investigations could help understand the mechanisms of seasonal influence in vaccine-induced responses.
\end{abstract}

\section{Introduction}

Over the last century, vaccination successfully reduced the disease burden of the more severe vaccine-preventable diseases (VPDs), such as diphtheria, pertussis, tetanus and poliomyelitis [1]. In the Netherlands, the majority of vaccines offered by the National Immunization Programme (NIP) cover more than $90 \%$ of the target population (children aged 0-19 years), following an age-based administration schedule [2].

The magnitude of antibody levels following vaccination varies between persons due to several factors: gender [3], age at vaccine inoculation [4], pre-existing antibody levels [5], health status [6] and immune system cycles [7].

Seasonal patterns in immunity, for example, cytokine profiles [8], or gene expression patterns [9], could affect the quality of the response to a vaccine. Through competition or enhancement, arms of the immune response influence each other in a possibly seasonal way. Seasonal differences in vaccine response may be explained by: variation in dose or duration of UV exposure, which may alter immune system and host resistance [10]; higher infection rates in certain seasons [11], which could possibly 'prime' the immune system producing an altered vaccine-induced response. Factors probably less important in the Netherlands are reduction in food availability, which impacts maternal and child's nutritional status and child's vaccine response [12], and pronounced differences in average temperatures throughout the year that may impact the vaccine cold chain and possibly vaccine response [13].

Past studies focused on single pathogens, had short follow-up and were mostly carried out in non-temperate climate zones. Two studies in temperate climate reported no association: in the Netherlands, immune responses to hepatitis B vaccine in college students were not associated with season of vaccination, nor were rubella and measles antibody levels following vaccination in children [14]. Inconclusive seasonal patterns were reported for immune response to hepatitis B vaccine in Austria [15].

The current exploratory study aims to investigate whether season of vaccination is associated with strength of the antibody response against a selection of pathogens in a representative nationwide sample of children from the Netherlands. 


\section{Methods}

\section{Study design and setting}

Serology was obtained from the Pienter- 2 study, a national crosssectional serosurveillance study performed between February 2006 and December 2007 in the Netherlands. In brief, Pienter-2 assessed the population's immune status against VPDs covered by the NIP through blood samples and self-administered questionnaires. Dutch inhabitants from 0 to 79 years old were included in the survey in two samples. A nationwide sample of 6348 participants, including an oversample of non-western migrants $(n=646)$, and a sample of 1518 participants from low vaccination coverage (LVC) areas (1517). A full description can be found elsewhere $[16,17]$.

\section{Selection of vaccine-preventable diseases}

We included a number of VPDs covered by the Dutch NIP in 2006/2007: diphtheria, tetanus and poliomyelitis, covered by the combination vaccine DP(a)T-IPV-Hib; measles, mumps and rubella, covered by the MMR combination vaccine; and meningococcal $\mathrm{C}$ disease, covered by the monovalent MenC vaccine.

Four VPDs covered by the NIP were not included: pertussis (due to the replacement of whole cell vaccine by acellular vaccine within the study period) [18]; pneumococcal disease (due to insufficient data, since the vaccine was implemented in 2006) [19]; Haemophilus influenzae type b (due to an increase in vaccine failure in 2002) [20] and hepatitis B (since it was only offered to risk groups at the time of study) [21].

\section{Selection of age ranges and vaccines}

According to the NIP schedule, vaccines are administered at distinct moments, either alone or in combination vaccines, and in a different number of doses (Fig. 1). Therefore, specific criteria applied to the selection of age ranges for the study of vaccineinduced immunity for each VPD (Fig. 2). When more than one vaccination was required to protect a child against a VPD, we considered the determining vaccination moment, the one in which the largest difference between pre- and post-vaccination antibody levels was observed (Guy Berbers, personal communication), as described in the literature [22]. Therefore, for DT-IPV, the season of the fourth vaccine dose given (at 11 months of age) was selected; for MMR, the season of the first vaccine dose (at 14 months of age) was considered.

The children in the study received the DT(a)P-IPV-Hib vaccine (Infanrix-IPV + Hib (GSK) or Infanrix Hexa (GSK) or Pediacel (SP-MDS)), the MMR vaccine (M-M-R VaxPro (SP-MDS)) and the MenC vaccine (NeisVac-C (Baxter)), according to the NIP schedule [2]. Vaccination dates were determined based on data from the nationwide information system Praeventis.

The concentrations of vaccine antibodies were assessed with the multiplex immunoassay (MIA). For diphtheria and tetanus, antibodies were determined by pentaplex MIA [23]. For poliomyelitis, anti-polio titres were measured via a standard neutralisation test [24]. For the simultaneous detection of antibodies against measles, mumps and rubella, a fluorescent bead-based MIA was performed [25]. Finally, the concentrations of MenC antibodies were determined using a fluorescent-bead-based MIA [26].

The cut-off values taken as seroconversion thresholds were: $0.1 \mathrm{IU} / \mathrm{ml}$ (full protection) and $0.01 \mathrm{IU} / \mathrm{ml}$ (basic immunity) for diphtheria [27], $0.1 \mathrm{IU} / \mathrm{ml}$ (full protection) and $0.01 \mathrm{IU} / \mathrm{ml}$ (basic immunity) for tetanus [28], 1:8 for the three polio types $[29,30], 0.2 \mathrm{IU} / \mathrm{ml}$ for measles [31, 32], $45 \mathrm{RU} / \mathrm{ml}$ for mumps [33], $10 \mathrm{IU} / \mathrm{ml}$ rubella [33] and $2 \mu \mathrm{g} / \mathrm{ml}$ for MenC [34-36] (Supplementary Table S1).

\section{Selection of sub-samples}

In the current study, the exclusion criteria (Fig. 2) for all respondents were: missing information on sampling date, missing serology data and being born before 1996 (i.e. adolescent or adult at study sampling period). Along with exclusion due to missing vaccination date, additional specific exclusion criteria applied to each (combination) vaccine, therefore three subsamples were created: DT-IPV, MMR and MenC, with possible multiple-occurrence of participants across sub-samples.

For the DT-IPV sub-sample, we excluded children who had not received exactly four DT(a)P-IPV-Hib vaccine doses (recommended, but not necessarily at 2, 3, 4 and 11 months of age). Children who had not received four vaccine doses or were younger than 9 months old and children who had received the booster dose at about 4 years of age or were older than 4.5 years old, were also excluded.

For the MMR sub-sample, we excluded children who had not received exactly one MMR vaccine dose (recommended, but not necessarily at 14 months of age); children receive a second dose MMR at 9 years of age, giving a relatively large time window for study.

For the MenC sub-sample, unvaccinated children, participants who were born before the MenC mass catch-up campaign (November 2002), and children with multiple MenC vaccinations were excluded.

In LVC areas, there was some circulation of MMR pathogens around or during the sampling period [37-39], and a relatively high incidence of meningococcal $\mathrm{C}$ disease before the introduction of the vaccine in 2002 [40]. We therefore excluded children from the LVC areas - potentially more exposed to the abovementioned VPDs [41] - from the analyses.

\section{Selection of variables}

\section{Dependent and independent variables}

Serum antibody levels (vaccine antigen-specific antibody) were measured following previously described laboratory methods for the Pienter-2 serosurveillance study [23-26]. Season of vaccination was defined as the quarter of the year in which the child was vaccinated (spring: April-June; summer: July-September; autumn: October-December and winter: January-March).

\section{Covariates}

The following covariates were included in the multivariable analysis: time post-vaccination, to account for possible waning antibody levels over time; and sex, considering sex-based differences in immune responses following vaccination [3]. Time postvaccination was included as a continuous variable, measured as months between vaccination moment and serum sampling.

\section{Statistical analysis}

Descriptive analyses were performed for population characteristics. Antibody levels were log-transformed or $\log 2$-transformed (poliovirus antibody levels), to normalise the data. Geometric mean titres/concentrations (GMT/C) with $95 \%$ confidence 


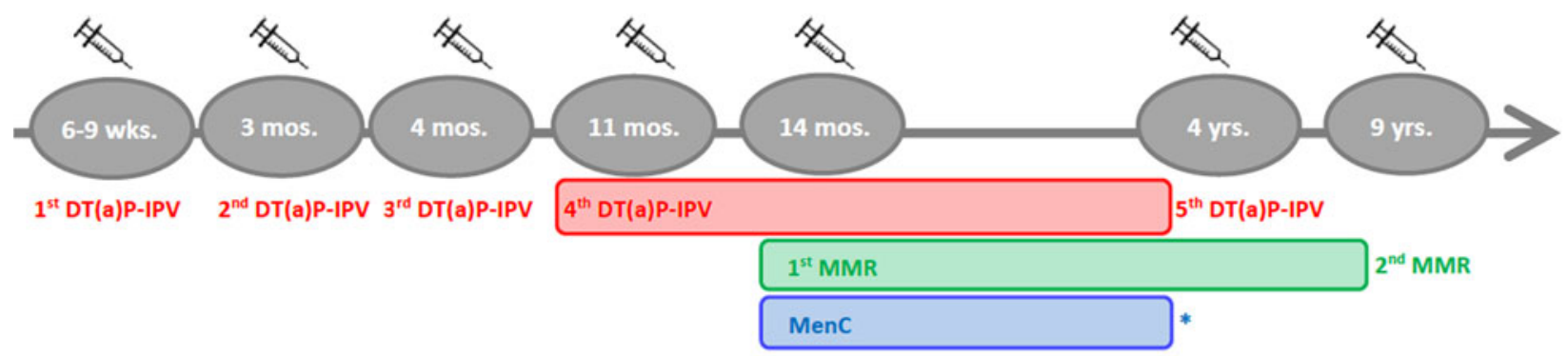

Vaccination moment

Children born before the introduction of MenC vaccine in the National Immunisation Programme (2002) were not included.

Fig. 1. Timeline of the vaccination schedule of the Dutch National Immunisation Programme for diphtheria, tetanus, poliomyelitis, measles, mumps, rubella and meningococcal C disease at the time of the Pienter-2 study (2006/2007). Coloured boxes indicate age range selected for the study of sub-samples.

intervals (95\% CI) and seroconversion rates were calculated. Pairwise Pearson correlation between antibody levels against different pathogens within individuals was performed along with Bonferroni correction for multiple testing. Differences in $\log ($ antibody levels) between seasons of vaccination were tested with linear multivariable regression analysis, using 'winter' and 'boy' as reference levels for season of vaccination and sex, respectively. The percentage difference in antibody levels was obtained by subtracting 1 from the exponential of the regression coefficient $\beta$ and multiplying it by 100: Difference $=\left[\left(e^{\beta}-1\right) \times 100\right]$.

Preliminary graphical analysis suggested sex differences in antibody levels according to season of vaccination and time postvaccination (Supplementary Fig. S1). Therefore, the full model was applied, with a three-way interaction term (season of vaccination $\times \operatorname{sex} \times$ time post-vaccination), and all lower-order interaction terms and main effects, for each pathogen. Backward model selection was performed. If including three-way interaction terms improved the fit of the model, the analysis was stratified by sex to ease the interpretation. 'Age at vaccination' was not included in the model due to little variance and predictive power. The significance level was set at 0.01 rather than 0.05 , to account for multiple testing.

$\mathrm{R}$ version 3.5.1 (R Development Core Team) and packages 'Im' and 'lme4' were used to analyse the data.

\section{Sensitivity analysis}

In the Pienter-2 study, non-Western migrants are oversampled, and thus this group is overrepresented in our sub-samples. This group might have higher antibody levels [24], possibly due to genetic differences and/or re-exposure to pathogens during visits to the country of origin. Therefore, we performed a sensitivity analysis on the national sample only, excluding oversampled non-Western migrant children.

To account for possible non-linearity of $\log$ (antibody) decay over time - associated, for example, with a vaccine eliciting two pools of antibodies with different decay rates -, an alternative model replacing the variable time post-vaccination by (log)time post-vaccination was also fitted. Goodness-of-fit was compared with that of the final model selected for each VPD.

\section{Results}

Of the 7865 respondents in the Pienter-2 study, covering all age groups, 2080 were children with information about sampling date (Fig. 2). After application of the sub-samples exclusion criteria, 434 children with a 'follow-up' period of about 45 months were eligible for the DT-IPV sub-sample, 811 for the MMR subsample with a 'follow-up' period of about 100 months and 311 for the MenC sub-sample with a 'follow-up' period of about 36 months (Figs 1 and 2). The sub-samples' characteristics are presented in Table 1. Distribution of children per group (national sample, oversampled children with non-Western background and, for DT-IPV sub-sample only, individuals from LVC areas) did not differ across seasons $(P>0.1)$. Median age at vaccination and time post-vaccination were relatively consistent across seasons of vaccination for all sub-samples, except for time postvaccination for the DT-IPV sub-sample $(P=0.018)$, which varied by as much as 8 months (summer vs. spring vaccination).

\section{Geometric mean titre/concentration (GMT/C) and seroconversion rates}

Table 2 shows the GMT/C (95\% CI) for each pathogen, per season of vaccination and sex. Differences in GMT/C between boys and girls were statistically significant at $\alpha=0.01$ level for mumps and rubella. Seroconversion rates, applying the thresholds given for each pathogen (Supplementary Table S1) did not vary by season of vaccination, except for the rubella vaccine, where rates varied between $90 \%$ and $99 \%(P=0.010)$, being lowest in children vaccinated in autumn and highest in winter.

\section{Fitted regression}

Season-stratified scatterplots of antibody levels against time postvaccination and the fitted regression lines from the final models are displayed in Supplementary Figure S1. The model fit of tetanus and poliovirus serotype 1 was improved when a three-way interaction term was included, therefore the analyses for these pathogens were stratified by sex. The lines show a decline in antibody titres against diphtheria, tetanus, poliovirus and MenC with time since vaccination.

\section{Season of vaccination and antibody levels}

No association between season of vaccination and antibody levels was found for any of the three poliovirus serotypes, for diphtheria, and for tetanus in girls (at $\alpha=0.01$ level, Table 3). In boys, the decline of tetanus antibodies after vaccination in summer was $6 \%$ per month slower than after vaccination in winter (Table 3: 
T. C. Abreu et al.

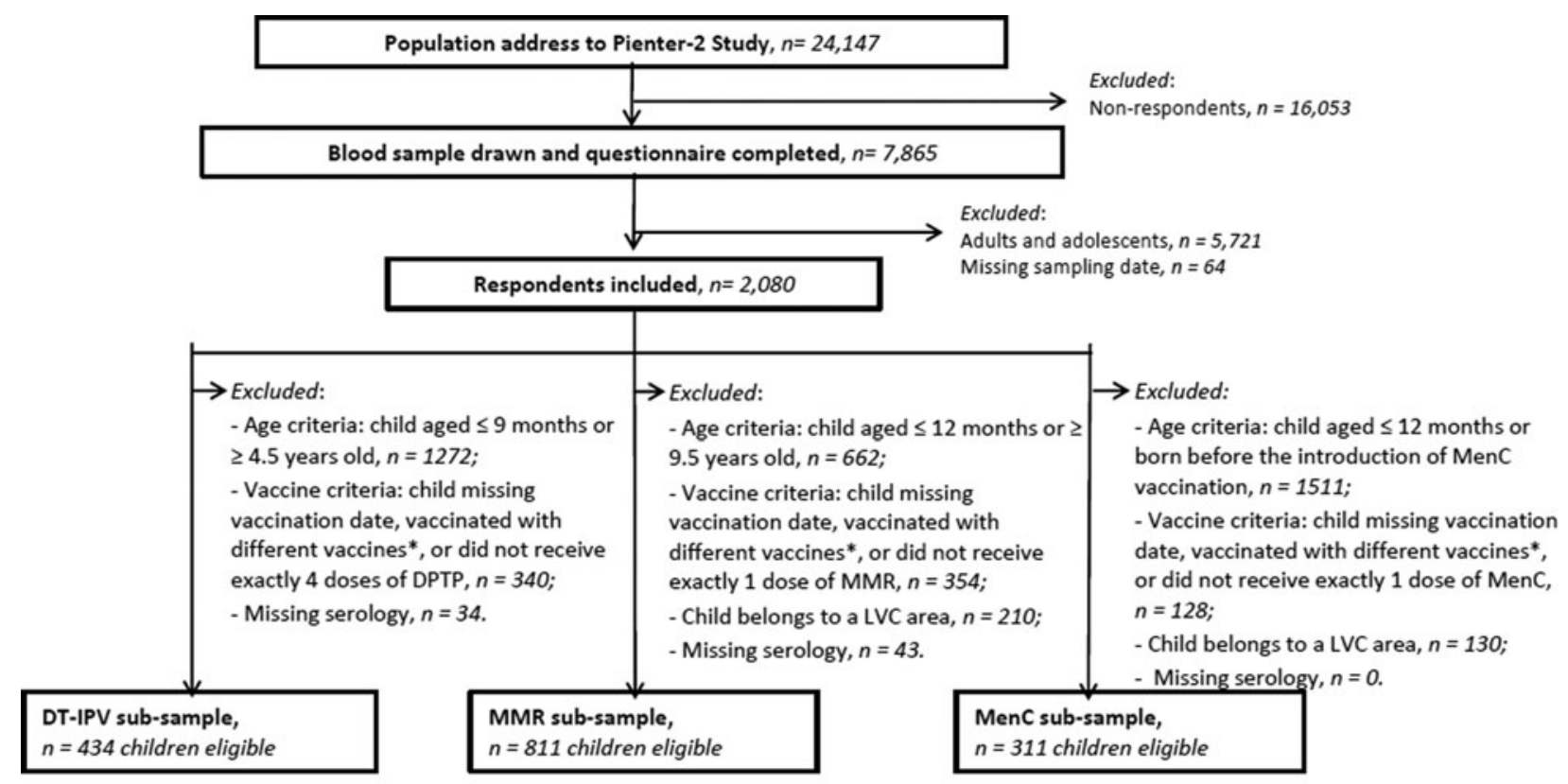

- Different vaccines: vaccines with different vaccine formulations, different combinations of components, or a vaccine component administered separately rather them in the combination vaccine recommended by the National Immunisation Programme for a given ages.

Fig. 2. Flow chart of exclusion/inclusion criteria, Pienter-2 study.

Table 1. Distribution of sample characteristics per season of vaccination, Pienter-2 study

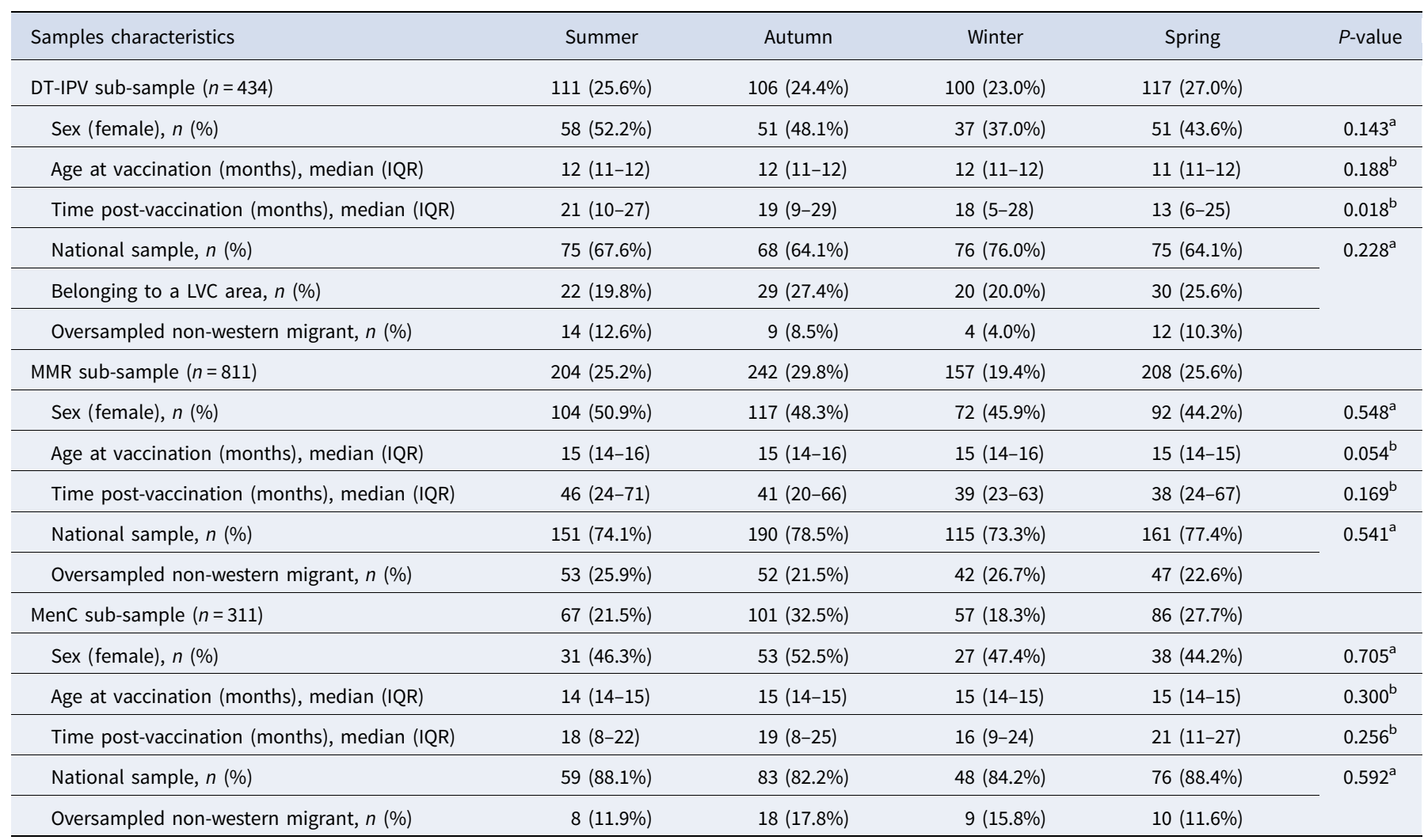

IQR, interquartile range.

${ }^{a}$ Pearson Chi-square test.

${ }^{\mathrm{b}}$ Kruskal-Wallis test. 
Table 2. Geometric mean titres/concentrations $(95 \% \mathrm{Cl})$, per season of vaccination and sex, Pienter-2 study

\begin{tabular}{|c|c|c|c|c|c|}
\hline & Winter & Spring & Summer & Autumn & $P$-value (overall mean sex difference) ${ }^{a}$ \\
\hline Diphtheria (IU/ml) & $0.13(0.09-0.19)$ & $0.12(0.09-0.17)$ & $0.08(0.06-0.10)$ & $0.10(0.08-0.13)$ & \\
\hline Boys & $0.14(0.09-0.21)$ & $0.13(0.08-0.20)$ & $0.08(0.05-0.11)$ & $0.09(0.06-0.13)$ & 0.848 \\
\hline Girls & $0.12(0.06-0.24)$ & $0.12(0.07-0.20)$ & $0.08(0.06-0.12)$ & $0.12(0.09-0.18)$ & \\
\hline Tetanus (IU/ml) & $0.83(0.59-1.16)$ & $0.91(0.68-1.21)$ & $0.63(0.49-0.82)$ & $0.80(0.63-1.03)$ & \\
\hline Boys & $0.81(0.52-1.26)$ & $1.02(0.69-1.50)$ & $0.61(0.44-0.85)$ & $0.77(0.55-1.08)$ & 0.798 \\
\hline Girls & $0.86(0.49-1.51)$ & $0.78(0.50-1.22)$ & $0.66(0.45-0.97)$ & $0.84(0.57-1.24)$ & \\
\hline Polio type 1 ( $\log 2$ GMT) & $6.88(6.29-7.47)$ & $7.31(6.74-7.87)$ & $6.91(6.42-5.93)$ & $6.49(5.95-7.03)$ & \\
\hline Boys & $6.83(6.09-7.57)$ & $7.32(6.52-8.11)$ & $6.25(5.58-6.91)$ & $5.62(4.90-6.33)$ & 0.068 \\
\hline Girls & $6.97(5.94-8.01)$ & $7.29(6.47-8.12)$ & $6.59(5.86-7.32)$ & $7.43(6.68-8.19)$ & \\
\hline Polio type $2(\log 2 \mathrm{GMT})$ & $7.33(6.70-7.96)$ & $7.60(7.01-8.19)$ & $6.54(5.99-7.09)$ & $6.83(6.26-7.40)$ & \\
\hline Boys & $7.06(6.26-7.86)$ & $7.79(6.95-8.63)$ & $6.08(5.35-6.80)$ & $5.98(5.16-6.80)$ & 0.034 \\
\hline Girls & $7.78(6.73-8.84)$ & $7.35(6.51-8.19)$ & $6.97(6.15-7.79)$ & $7.75(7.01-8.48)$ & \\
\hline Polio type $3(\log 2$ GMT) & $6.04(5.31-6.77)$ & $6.43(5.69-7.16)$ & $5.05(4.40-5.69)$ & $5.56(4.87-6.24)$ & \\
\hline Boys & $6.35(5.43-7.27)$ & $6.71(5.67-7.75)$ & $4.28(3.45-5.12)$ & $4.58(3.68-5.48)$ & 0.232 \\
\hline Girls & $5.51(4.25-6.77)$ & $6.06(5.01-7.11)$ & $5.74(4.79-6.70)$ & $6.61(5.61-7.60)$ & \\
\hline Measles (IU/ml) & $1.89(1.56-2.29)$ & $1.72(1.48-2.01)$ & $1.64(1.42-1.89)$ & $1.63(1.40-1.90)$ & \\
\hline Boys & $1.89(1.48-2.41)$ & $1.58(1.27-1.96)$ & $1.54(1.24-1.92)$ & $1.60(1.30-1.97)$ & 0.279 \\
\hline Girls & $1.88(1.38-2.57)$ & $1.93(1.56-2.38)$ & $1.74(1.43-2.11)$ & $1.66(1.32-2.09)$ & \\
\hline Mumps (RU/ml) & $125(102-152)$ & $119(103-137)$ & $122(107-139)$ & $96(80-116)$ & \\
\hline Boys & $132(101-173)$ & $97(79-119)$ & $104(87-126)$ & $88(70-111)$ & 0.009 \\
\hline Girls & $117(86-159)$ & $154(128-186)$ & $142(119-170)$ & $106(78-143)$ & \\
\hline Rubella (IU/ml) & $76(65-89)$ & $58(50-68)$ & $55(47-65)$ & $47(39-56)$ & \\
\hline Boys & $76(63-92)$ & $48(39-60)$ & $46(35-60)$ & $39(30-51)$ & 0.001 \\
\hline Girls & $76(59-100)$ & $73(59-91)$ & $66(55-79)$ & $56(44-72)$ & \\
\hline MenC $(\mu \mathrm{g} / \mathrm{ml})$ & $0.66(0.44-0.99)$ & $0.80(0.58-1.12)$ & $0.77(0.55-1.09)$ & $0.95(0.73-1.24)$ & \\
\hline Boys & $0.74(0.48-1.15)$ & $0.80(0.51-1.24)$ & $0.71(0.44-1.16)$ & $0.75(0.54-1.04)$ & 0.328 \\
\hline Girls & $0.58(0.28-1.21)$ & $0.81(0.49-1.36)$ & $0.85(0.51-1.42)$ & $1.19(0.80-1.77)$ & \\
\hline
\end{tabular}

Note: Confidence intervals $(\mathrm{Cl})$ in parentheses.

${ }^{a} t$ test for overall mean difference between boys and girls.

$\beta=0.06 ; P<0.01$; Supplementary Fig. S1B). This decline led antibody concentrations to levels close to sub-protective concentrations by 40 months post-vaccination (by the time children receive a booster against tetanus) (Supplementary Fig. S1B). No association was found between season of vaccination and antibody levels induced against measles, mumps and rubella (Table 4 and Supplementary Fig. S1F-H), nor meningococcus C (Table 5 and Supplementary Fig. S1I).

When considering a less stringent significance level (at $\alpha=$ 0.05 level), we observed that children vaccinated in summer had lower antibody levels against diphtheria (36\% lower; $\beta=-0.45$; $P=0.029$ ), against poliovirus serotype 2 (51\% lower; $\beta=-0.72$; $P=0.048$ ), and against poliovirus serotype 3 (56\% lower; $\beta=$ $-0.83 ; P=0.045)$ compared to children vaccinated in winter. For boys, summer vaccination induced tetanus antibody levels directly post-vaccination $72 \%$ lower $(\beta=-1.28 ; P=0.013)$ in comparison with winter-vaccinated boys (Table 3 ). Children vaccinated in autumn had rubella antibody levels directly postvaccination $38 \%$ lower $(\beta=-0.48 ; P=0.031)$ compared to winter- vaccinated children. Autumn vaccination induced mumps antibody levels over the 3 -year 'follow-up' period $24 \%$ lower ( $\beta=$ $-0.27 ; P=0.032)$ than winter vaccination. The rate of rubella antibodies decline over time was $1 \%$ per month faster when children were vaccinated in summer $(\beta=-0.01 ; P=0.021)$ or spring ( $\beta=-0.01 ; P=0.032$ ), compared to winter vaccination (Table 4 ). Autumn vaccination induced MenC antibody levels over the 3 -year 'follow-up' period $57 \%$ higher $(\beta=0.45 ; P=0.030)$, when compared to winter vaccination (Table 5).

\section{Sex differences}

The antibody response induced against polio type 2 was overall higher in girls than in boys (Table 3: $\beta=0.72 ; \quad P<0.01$; Supplementary Fig. S1D). Furthermore, overall antibody levels against mumps and rubella were higher in girls than in boys (Table 4: $\beta=0.23$ and $\beta=0.32$, respectively; $P<0.01$ ), but not for measles. This is also visualised in Supplementary Figure S1F-H. 
Table 3. Linear multivariate regression models estimates for antibody levels as a function of season of vaccination, time post-vaccination and sex for DT-IPV sub-sample, Pienter-2 study

\begin{tabular}{|c|c|c|c|c|c|c|c|}
\hline & \multirow{2}{*}{ Diphtheria $^{a}$} & \multicolumn{2}{|c|}{ Tetanus $^{\mathrm{a}}$} & \multicolumn{2}{|c|}{ Polio type $1^{\mathrm{b}}$} & \multirow{2}{*}{ Polio type $2^{\mathrm{b}}$} & \multirow{2}{*}{ Polio type $3^{\mathrm{b}}$} \\
\hline & & Girls & Boys & Girls & Boys & & \\
\hline Intercept & $\begin{array}{l}-1.07^{\star \star}(-1.45 \text { to } \\
0.68)\end{array}$ & $1.09^{\star \star}(0.55-1.64)$ & $1.30^{\star \star}(0.73-1.86)$ & $9.20^{\star \star}(8.17-10.24)$ & $8.87^{\star \star}(8.04-9.71)$ & $9.72^{\star \star}(9.04-10.40)$ & $9.41^{\star \star}(8.63-10.18)$ \\
\hline Winter vaccination & ref. & ref. & ref. & ref. & ref. & ref. & ref. \\
\hline Spring vaccination & $-0.23(-0.63$ to 0.16$)$ & $\begin{array}{l}-0.28(-0.85 \text { to } \\
0.29)\end{array}$ & $\begin{array}{l}-0.14(-0.89 \text { to } \\
0.61)\end{array}$ & $\begin{array}{l}0.004(-1.08 \text { to } \\
1.09)\end{array}$ & $0.09(-0.81$ to 0.98$)$ & $\begin{array}{l}-0.22(-0.91 \text { to } \\
0.48)\end{array}$ & $\begin{array}{l}-0.23(-1.03 \text { to } \\
0.57)\end{array}$ \\
\hline Summer vaccination & $\begin{array}{l}-0.45^{\star}(-0.85 \text { to } \\
0.05)\end{array}$ & $\begin{array}{l}-0.33(-0.88 \text { to } \\
0.22)\end{array}$ & $\begin{array}{l}-1.28^{\star}(-2.27 \text { to } \\
0.28)\end{array}$ & $\begin{array}{l}-0.50(-1.55 \text { to } \\
0.56)\end{array}$ & $\begin{array}{l}-0.24(-1.18 \text { to } \\
0.70)\end{array}$ & $\begin{array}{l}-0.72^{\star}(-1.42 \text { to } \\
0.01)\end{array}$ & $\begin{array}{l}-0.83^{\star}(-1.64 \text { to } \\
0.02)\end{array}$ \\
\hline Autumn vaccination & $-0.23(-0.64$ to 0.18$)$ & $\begin{array}{l}-0.24(-0.81 \text { to } \\
0.33)\end{array}$ & $\begin{array}{l}-0.02(-0.96 \text { to } \\
0.92)\end{array}$ & $0.06(-1.02$ to 1.15$)$ & $\begin{array}{l}-0.81(-1.75 \text { to } \\
0.12)\end{array}$ & $\begin{array}{l}-0.53(-1.25 \text { to } \\
0.18)\end{array}$ & $\begin{array}{l}-0.48(-1.30 \text { to } \\
0.34)\end{array}$ \\
\hline $\begin{array}{l}\text { [1] Time } \\
\text { post-vaccination }{ }^{c}\end{array}$ & $\begin{array}{l}-0.05^{\star \star}(-0.07 \text { to } \\
0.04)\end{array}$ & $\begin{array}{l}-0.06^{\star \star}(-0.08 \text { to } \\
0.05)\end{array}$ & $\begin{array}{l}-0.09^{\star \star}(-0.11 \text { to } \\
0.06)\end{array}$ & $\begin{array}{l}-0.11^{\star \star}(-0.14 \text { to } \\
0.08)\end{array}$ & $\begin{array}{l}-0.12^{\star \star}(-0.15 \text { to } \\
0.09)\end{array}$ & $\begin{array}{l}-0.15^{\star \star}(-0.17 \text { to } \\
0.12)\end{array}$ & $\begin{array}{l}-0.20^{\star \star}(-0.22 \text { to } \\
0.17)\end{array}$ \\
\hline Girl & $0.07(-0.21$ to 0.36$)$ & - & - & - & - & $0.72^{\star \star}(0.23-1.22)$ & $0.54(-0.03$ to 1.11$)$ \\
\hline Winter vaccination $\times[1]$ & - & - & ref. & - & - & - & - \\
\hline Spring vaccination $\times[1]$ & - & - & $0.01(-0.03$ to 0.04$)$ & - & - & - & - \\
\hline $\begin{array}{l}\text { Summer vaccination } \times \\
{[1]}\end{array}$ & - & - & $0.06^{\star \star}(0.02$ to 0.11$)$ & - & - & - & - \\
\hline $\begin{array}{l}\text { Autumn vaccination } \times \\
\text { [1] }\end{array}$ & - & - & $0.01(-0.03$ to 0.06$)$ & - & - & - & - \\
\hline Observations & 434 & 197 & 237 & 197 & 237 & 434 & 434 \\
\hline Adjusted $R^{2}$ & 0.14 & 0.21 & 0.28 & 0.20 & 0.22 & 0.29 & 0.35 \\
\hline Residual standard error & $1.48(\mathrm{df}=428)$ & $1.34(\mathrm{df}=192)$ & $1.26(\mathrm{df}=229)$ & $2.56(\mathrm{df}=192)$ & $2.56(\mathrm{df}=232)$ & $2.60(\mathrm{df}=428)$ & $2.98(\mathrm{df}=428)$ \\
\hline$F$ statistic & $15.37^{\star \star}(d f=5 ; 428)$ & $14.01^{\star \star}(\mathrm{df}=4 ; 192)$ & $14.19^{\star \star}(\mathrm{df}=7 ; 229)$ & $13.07^{\star \star}(\mathrm{df}=4 ; 192)$ & $17.60^{\star \star}(\mathrm{df}=4 ; 232)$ & $36.65^{\star \star}(\mathrm{df}=5 ; 428)$ & $48.28^{\star \star}(\mathrm{df}=5 ; 428)$ \\
\hline
\end{tabular}

Notes: Confidence intervals $(95 \% \mathrm{Cl})$ in parentheses; reference levels are winter (season of vaccination) and boy (sex).

Log-transformed.

Log 2-transform 
Table 4. Linear multivariate regression model estimates for antibody levels as a function of season of vaccination, time post-vaccination and sex for MMR sub-sample, Pienter-2 study

\begin{tabular}{|c|c|c|c|}
\hline & Measles $^{a}$ & Mumps $^{a}$ & Rubella $^{a}$ \\
\hline Intercept & $1.10^{\star \star}(0.88-1.32)$ & $4.86^{\star \star}(4.61-5.10)$ & $4.59^{\star \star}(4.24-4.95)$ \\
\hline Winter vaccination & ref. & ref. & ref. \\
\hline Spring vaccination & $-0.07(-0.30$ to 0.16$)$ & $-0.04(-0.29$ to 0.21$)$ & $0.18(-0.27$ to 0.64$)$ \\
\hline Summer vaccination & $-0.09(-0.32$ to 0.14$)$ & $-0.02(-0.27$ to 0.24$)$ & $0.22(-0.25$ to 0.68$)$ \\
\hline Autumn vaccination & $-0.15(-0.38$ to 0.07$)$ & $-0.27^{\star}(-0.51$ to 0.02$)$ & $-0.48^{\star}(-0.92$ to 0.04$)$ \\
\hline [1] Time post-vaccination ${ }^{b}$ & $-0.01^{\star \star}(-0.01$ to 0.01$)$ & $-0.003^{\star}(-0.01$ to 0.0001$)$ & $-0.01^{\star \star}(-0.02$ to 0.002$)$ \\
\hline Girl & $0.11(-0.04$ to 0.26$)$ & $0.23^{\star \star}(0.07-0.40)$ & $0.32^{\star \star}(0.16-0.48)$ \\
\hline Winter vaccination $\times[1]$ & - & - & ref. \\
\hline Spring vaccination $\times[1]$ & - & - & $-0.01^{\star}(-0.02$ to 0.001$)$ \\
\hline Summer vaccination $\times[1]$ & - & - & $-0.01^{\star}(-0.02$ to 0.002$)$ \\
\hline Autumn vaccination $\times[1]$ & - & - & $-0.0005(-0.01$ to 0.01$)$ \\
\hline Observations & 811 & 811 & 811 \\
\hline Adjusted $R^{2}$ & 0.07 & 0.02 & 0.14 \\
\hline Residual standard error & $1.11(\mathrm{df}=805)$ & $1.21(\mathrm{df}=805)$ & $1.13(\mathrm{df}=802)$ \\
\hline F statistic & $14.10^{\star \star}(\mathrm{df}=5 ; 805)$ & $3.54^{\star \star}(\mathrm{df}=5 ; 805)$ & $17.97^{\star \star}(\mathrm{df}=8 ; 802)$ \\
\hline
\end{tabular}

Notes: Confidence intervals $(95 \% \mathrm{Cl})$ in parentheses; reference levels are winter (season of vaccination) and boy (sex).

aLog-transformed.

bIn months.

Levels of significance: ${ }^{\star \star} P<0.01 ;{ }^{\star} P<0.05$.

Table 5. Linear multivariate regression model estimates for antibody levels as a function of season of vaccination, time post-vaccination and sex for MenC sub-sample, Pienter-2 study

\begin{tabular}{ll}
\hline & \multicolumn{1}{c}{ MenC $^{\mathrm{a}}$} \\
\hline Intercept & $0.69^{\star \star}(0.27-1.10)$ \\
\hline Winter vaccination & ref. \\
\hline Spring vaccination & $0.41(-0.004$ to 0.83$)$ \\
\hline Summer vaccination & $0.16(-0.28$ to 0.60$)$ \\
\hline Autumn vaccination & $0.45^{\star}(0.05-0.85)$ \\
\hline Time post-vaccination ${ }^{\mathrm{b}}$ & $-0.07^{\star \star}(-0.09$ to 0.06$)$ \\
\hline Girl & $0.20(-0.08$ to 0.48$)$ \\
\hline Observations & 311 \\
\hline Adjusted $R^{2}$ & 0.26 \\
\hline Residual standard error & $1.24(\mathrm{df}=305)$ \\
\hline F statistic & $22.33^{\star \star}(\mathrm{df}=5 ; 305)$ \\
\hline
\end{tabular}

Notes: Confidence intervals $(95 \% \mathrm{Cl})$ in parentheses; reference levels are winter (season of vaccination) and boy (sex).

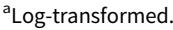

b In months.

Levels of significance: ${ }^{\star \star} P<0.01 ;{ }^{\star} P<0.05$.

\section{Correlation of antibody levels against different pathogens within individuals}

The antibody levels elicited by the different components of combination vaccines correlated moderately within children: the pairwise Pearson correlation of antibody levels induced by MMR and DT-IPV combination vaccines was $\geqslant 0.39$ and $\geqslant 0.41$, respectively $(P \leqslant 0.001)$. However, the antibody levels elicited by different vaccines administered on the same day (i.e. MMR and MenC) showed a different pattern: MenC levels did not correlate with measles $(r=0.12, P=0.03)$, mumps $(r=0.08, P=0.15)$ or rubella antibody levels $(r=0.14, P=0.01)$ (Supplementary Table S2).

\section{Sensitivity analyses}

The sensitivity analysis excluding oversampled non-Western children (Supplementary Tables S3-S5) showed no seasonal association anymore. It yielded only one significant term at $\alpha=0.01$ level: rubella antibody levels were higher in girls than in boys ( $\beta=0.27, P<0.01$; Supplementary Table S4).

A model accounting for a possible biphasic decay of antibodies post-vaccination only improved the model fit to the diphtheria antibodies (adj. $R^{2}$ from 0.14 to 0.21 ), and to tetanus antibodies in boys (adj. $R^{2}$ from 0.28 to 0.31 ), compared with the best-fit models assuming a monophasic antibody decay (Supplementary Table S6). These models did not show a significant association between antibody levels and season of vaccination against diphtheria nor for tetanus (data not shown).

\section{Discussion}

We found little evidence to support the hypothesis that season of vaccination in children may be associated with different antibody levels for the diseases investigated in a nationwide cohort from the Netherlands. The only indication for a seasonal association at the conservative significance threshold of 0.01 was found for boys vaccinated against tetanus, who showed a slower decline in antibody levels after vaccination in summer compared to vaccination in winter. However, the association between season of vaccination and tetanus antibody levels seems to be better modelled when 
accounting for a biphasic decay of antibodies post-vaccination, where the association is then no longer present. At a less conservative significance level $(P$-value $<0.05)$, we found some tendency of association between season of vaccination and antibody levels for all the diseases investigated - except for poliovirus serotype 1 and measles - with associations for mumps, rubella and diphtheria having a $P$-value around 0.03 .

To our knowledge, our study is the first to investigate the impact of season of vaccination on antibodies against mumps and MenC. Moreover, no studies have focused on interaction effects involving season of vaccination, sex and time postvaccination.

A study by Moore et al. did not find an association between season of vaccination and tetanus and diphtheria antibody levels in a cohort of 138 infants in The Gambia [42]. Antibody levels were measured and compared immediately before the two first doses of tetanus and diphtheria vaccines were administered to children at 8 and 16 weeks of age. We investigated immunity in older children, over a broader age-range, hampering comparisons.

Consistent with a report from the Netherlands [14], season of vaccination did not influence measles antibody levels in our study. In a study in Guinea-Bissau, however, in a cohort of 415 children, those vaccinated during the rainy season at 9 months of age had higher measles antibody levels at 24 months of age than those vaccinated in the dry season [43]. In a study with 203 children aged 4-5 years, Linder et al. found a stronger immune response to rubella vaccine at 12 months in children vaccinated in winter in Israel [44]. On the other hand, the previous report in the Netherlands could not establish differences in rubella antibody levels related to season of vaccination at 14 months, in a cohort of 719 children aged 2-7 years old [14], just as in our study.

\section{Correlation of antibody levels against different pathogens within individuals}

Although MMR and MenC vaccines are administered to children on the same day, the individual's antibody levels did not show similar immunogenicity patterns by season of vaccination. Differences in the magnitude of responses induced by different vaccines administered on the same day (i.e. same season) confirm the idea that the response - and potentially the effect of seasonality - is antigen-specific and is not linked to an individual's capacity to mount/produce an immune response. When present, the impact of seasonality on the vaccine-induced response seems to be pathogen-specific, i.e. the magnitude of the response elicited by some vaccines seems to be more affected by the seasonal effect than others. Different pathogens engage the immune response through different pathways, which may themselves be differentially affected by season.

\section{Sensitivity analyses}

The seasonal effect found for boys vaccinated against tetanus was no longer statistically significant when we performed sensitivity analysis excluding the oversampled children: this could result from a loss of power due to smaller sample size, or it might indicate that non-Western children are subject to a stronger seasonal effect, for instance due to re-exposure to pathogens when travelling to their homeland, different behaviour regarding sun light exposure, or genetic differences impacting their immune function $[45,46]$. This is in line with the higher GMT/C found in oversampled children for all antibody levels (except for measles), when compared to the levels of the national sample (data not shown), and as previously reported in the non-Western community in the Netherlands [24].

We assumed a linear decay of log(antibody levels), which could lead to over- or underestimation of model estimates if in fact vaccination would induce different antibody populations with varying decay rates [47]. The latter might entail a biphasic decay of $\log$ (antibody levels). Therefore, we compared the model fit of the linear models with an alternative model including log-transformed 'time post-vaccination', which showed that only the model fit of diphtheria and tetanus (boys) benefited from the inclusion of the non-linear independent variable. It is interesting to note that the linear model for which we found the strongest association between antibody levels and season of vaccination (tetanus antibody decline over time in boys) might be better modelled with a biphasic decay, and therefore may no longer present that association. Nevertheless, it is fair to assume a linear antibody decay for the vaccines that did not have their model fit improved by the inclusion of a biphasic decay of $\log$ (antibody levels). Thus, linear models that showed tendencies of association between season of vaccination and immunity (i.e. $P$-value $<0.05$ ) could still be used and might produce increased significance levels with a larger sample size.

\section{Variables not controlled for}

We did not control for maternal antibody status, since children older than 9 months (the minimum age included in our subsamples) are likely no longer protected by maternal antibodies [48]. Moreover, despite the fact that infant age may largely influence the immune response to a vaccine [4], age at vaccination had small variance and low predictive power in our dataset, and its inclusion in the models could cause precision issues and poor performance. This limited variability in timing of vaccination means we cannot disentangle the association between season of vaccination from the association with season of birth.

Even though some literature suggests that vaccine administration timing (e.g. morning or afternoon) might impact the induced immune response [7], we do not believe that this effect played a role in our study, since vaccines were routinely randomly administered throughout the day, and therefore, vaccine administration timing is not likely to vary across seasons.

Our results were not corrected for socioeconomic status, which could be a proxy for factors that might influence antibody levels, such as nutritional status. Nonetheless, nutritional status is not likely to vary by season of vaccination in children in the Netherlands, as opposed to countries where the supply of food is reduced in some periods of the year.

\section{Limitations}

A limitation of our analysis is the cross-sectional nature of the study, which does not control for the influence of individual heterogeneity in antibody waning rates. We compared the decay rates obtained in the current study at the population level with estimated waning rates of MMR antibody levels in a longitudinal cohort study of 43 children from the Netherlands over 3 years [49]. The decline in rubella antibody levels was the only waning rate comparable to our study (Supplementary Table S7; $\beta_{\text {time post-vaccination }}=-0.01 \mathrm{ln} \mathrm{IU} / \mathrm{ml}$ per month after vaccination, for both studies). The waning rate for measles antibody levels was not statistically significant and mumps antibody levels 
showed an increase over time (Supplementary Table S7; $\beta_{\text {time post-vaccination }}=0.02 \mathrm{ln} \mathrm{IU} / \mathrm{ml}$ per month after vaccination) in the longitudinal study. It is noteworthy that a booster dose of DT(a)P-IPV vaccine is recommended at 4 years old, which is around the latest longitudinal sampling moment in the above cohort study and could have non-specifically enhanced measles and mumps antibody levels, as this was also observed for other vaccines [50]. We believe that the same effect would not impact our main findings, as a large range of age groups was sampled. In addition, participants of the longitudinal study might have been heterogeneously exposed to measles virus, since most of them were born during or shortly before a measles outbreak in the Netherlands in 2013 [51], or ultimately, the sample size did not provide sufficient statistical power to estimate the waning rates. Nevertheless, considering that our cross-sectional observations followed a longitudinal pattern and that studies observed comparable trends in immune response per time after exposure in cross-sectional and longitudinal data (individual basis) [52], we believe that our cross-sectional design provides reasonable estimates of waning antibody rates.

We assumed the environmental exposure to pathogens was minimal, based on national notification systems and surveillance information. Moreover, we excluded children from areas where certain pathogens were more likely to circulate. It is noteworthy that vaccine responses against pathogens not circulating extensively in the environment (e.g. diphtheria and poliomyelitis in the Netherlands) may be suitable biomarkers for analysis of seasonality of immunity, since the seasonal effect of the vaccine on antibody levels is unlikely to be influenced by the effect of seasonal circulation of the pathogen.

We also assumed that differences in the ambient temperature did not affect the vaccines' cold chain, as it could have been the case in countries with higher average yearly temperatures.

Although for tetanus, diphtheria, poliomyelitis, measles and rubella, antibody levels are good correlates of protection, and MenC IgG antibody levels correlate with the 'gold standard' (serum bactericidal assay), mumps antibodies poorly correlate with immunological protection [53]. Therefore, differences in mumps antibody levels, whether related to season of vaccination or not, may not result in altered immunological protection.

It is known that the length of time between vaccine doses impacts the antibody production following the latest one [54], and thus when multiple doses are given within a brief period, teasing out the contribution of each dose in shaping the magnitude of the immune response is practically unattainable. Therefore, although we selected the vaccination moment at which the largest variation between pre- and post-vaccination antibody levels occurs, we were unable to disentangle the impact of the season of the previous DT-IPV doses on children's antibody levels.

In conclusion, our findings provide limited evidence that season of vaccination in children may be associated with antibody levels against the different pathogens investigated in a nationwide cohort from the Netherlands. Moreover, antibody levels do not seem to fall below protection levels over the study period considered, so clinical implications might be limited. We have applied a conservative significance threshold of 0.01 ; however, it is noteworthy that associations between season of vaccination and immunity against mumps, rubella and diphtheria had a $P$-value around 0.03 . Possibly, adding the data obtained in the last nationwide serological study in the Netherlands (Pienter-3) could provide the power required to increase significance level of the results. Thus, linear models that showed tendencies of association between season of vaccination and immunity (i.e. $P$-value $<0.05$ ) could still be used and might produce increased significance levels with a larger sample size. In addition, further longitudinal immunological and epidemiological investigation of seasonality of vaccine-induced immunity in countries of temperate climate is needed to confirm our findings, to assess clinical relevance and to help understand the possible underlying mechanisms involved.

Supplementary material. The supplementary material for this article can be found at https://doi.org/10.1017/S0950268820002691.

Acknowledgements. We thank Rob van Binnendijk and Iris Brinkman for providing the longitudinal cohort data on MMR immune response and for their insights on immunology. We also thank Axel Bonacic Marinovic for providing valuable advice on modelling antibody decay and Pieter van Gageldonk for providing advice on criteria for seroprevalence.

Financial support. This research did not receive any specific grant from funding agencies in the public, commercial or not-for-profit sectors.

Conflict of interest. None of the authors reported a conflict of interest.

Data availability statement. The data that support the findings of this study are available on request from the authors.

\section{References}

1. van Wijhe M et al. (2016) Effect of vaccination programmes on mortality burden among children and young adults in the Netherlands during the 20th century: a historical analysis. Lancet Infectious Diseases 16, 592-598.

2. van Lier EA et al. (2019) Vaccinatiegraad en jaarverslag Rijksvaccinatieprogramma Nederland 2018. Bilthoven, Netherlands: National Institute for Public Health and the Environment (RIVM).

3. Klein SL, Marriott I and Fish EN (2015) Sex-based differences in immune function and responses to vaccination. Transactions of the Royal Society of Tropical Medicine and Hygiene 109, 9-15.

4. Voysey M et al. (2017) The influence of maternally derived antibody and infant age at vaccination on infant vaccine responses: an individual participant meta-analysis. JAMA Pediatrics 171, 637-646.

5. Idoko OT et al. (2014) The impact of pre-existing antibody on subsequent immune responses to meningococcal A-containing vaccines. Vaccine 32, 4220-4227.

6. Eisenhut $\mathbf{M}$ et al. (2016) Antibody responses to immunizations in children with type I diabetes mellitus: a case-control study. Clinical and Vaccine Immunology 23, 873-877.

7. Long JE et al. (2016) Morning vaccination enhances antibody response over afternoon vaccination: a cluster-randomised trial. Vaccine 34, 2679-2685.

8. Horst RT et al. (2016) Host and environmental factors influencing individual human cytokine responses. Cell 167, 1111-1124.e1113.

9. Dopico XC et al. (2015) Widespread seasonal gene expression reveals annual differences in human immunity and physiology. Nature Communications 6, 7000 .

10. Termorshuizen F et al. (2002) A review of studies on the effects of ultraviolet irradiation on the resistance to infections: evidence from rodent infection models and verification by experimental and observational human studies. International Immunopharmacology 2, 263-275.

11. Turabian JL (2017) The variation of seasonal diseases in family medicine depends on infectious diseases and these are mainly respiratory diseases. Journal of General Practice 5, 1-6.

12. Obanewa $\mathbf{O}$ and Newell ML (2017) Maternal nutritional status during pregnancy and infant immune response to routine childhood vaccinations. Future Virology 12, 525-536.

13. Kartoglu U and Milstien J (2014) Tools and approaches to ensure quality of vaccines throughout the cold chain. Expert Review of Vaccines 13, 843-854. 
14. Termorshuizen F et al. (2001) Vaccine induced antibody responses in relation to season. Bilthoven, The Netherlands. RIVM report 640300004.

15. Rendi-Wagner $\mathbf{P}$ et al. (2001) Antibody-response to three recombinant hepatitis B vaccines: comparative evaluation of multicenter travel-clinic based experience. Vaccine 19, 2055-2060.

16. Mollema L et al. (2010) PIENTER 2-project: second research project on the protection against infectious diseases offered by the national immunization programme in the Netherlands. RIVM [230421001/2009].

17. van der Klis FR et al. (2009) Second national serum bank for populationbased seroprevalence studies in the Netherlands. Netherlands Journal of Medicine 67, 301-308.

18. de Greeff SC et al. (2008) Impact of acellular pertussis preschool booster vaccination on disease burden of pertussis in The Netherlands. Pediatric Infectious Disease Journal 27, 218-223.

19. Knol MJ et al. (2015) Invasive pneumococcal disease 3 years after Introduction of 10-valent pneumococcal conjugate vaccine, the Netherlands. Emerging Infectious Diseases 21, 2040-2044.

20. Breukels MA et al. (2001) Immunological characterization of conjugated Haemophilus influenzae type $\mathrm{b}$ vaccine failure in infants. Clinical Infectious Diseases 32, 1700-1705.

21. Houweling $\mathbf{H}$ et al. (2010) Public vaccination programmes against hepatitis B in The Netherlands: assessing whether a targeted or a universal approach is appropriate. Vaccine 28, 7723-7730.

22. Steens A et al. (2010) High tetanus antitoxin antibody concentrations in the Netherlands: a seroepidemiological study. Vaccine 28, 7803-7809.

23. van Gageldonk PG et al. (2008) Development and validation of a multiplex immunoassay for the simultaneous determination of serum antibodies to Bordetella pertussis, diphtheria and tetanus. Journal of Immunological Methods 335, 79-89.

24. van der Maas NA et al. (2014) Immunity against poliomyelitis in the Netherlands, assessed in 2006 to 2007: the importance of completing a vaccination series. Eurosurveillance 19, 20705.

25. Smits GP et al. (2012) Development of a bead-based multiplex immunoassay for simultaneous quantitative detection of IgG serum antibodies against measles, mumps, rubella, and varicella-zoster virus. Clinical and Vaccine Immunology 19, 396-400.

26. de Voer RM et al. (2009) Simultaneous detection of Haemophilus influenzae type b polysaccharide-specific antibodies and Neisseria meningitidis serogroup A, C, Y, and $\mathrm{W}-135$ polysaccharide-specific antibodies in a fluorescent-bead-based multiplex immunoassay. Clinical and Vaccine Immunology 16, 433-436.

27. World Health Organization (WHO) (2009) The Immunological Basis for Immunization Series: Module 2: Diphtheria. Geneva: World Health Organization (WHO).

28. World Health Organization (WHO) (2018) The Immunological Basis for Immunization Series: Module 3: Tetanus. Geneva: World Health Organization (WHO).

29. Asturias EJ et al. (2007) Randomized trial of inactivated and live polio vaccine schedules in Guatemalan infants. Journal of Infectious Diseases 196, 692-698.

30. World Health Organization (WHO) (1993) The Immunological Basis for Immunization Series: Module 6: Poliomyelitis. Geneva: World Health Organization (WHO).

31. Chen RT et al. (1990) Measles antibody: reevaluation of protective titers. Journal of Infectious Diseases 162, 1036-1042.

32. Christenson B and Böttiger M (1990) Methods for screening the naturally acquired and vaccine-induced immunity to the measles virus. Biologicals 18, 207-211.

33. Andrews $\mathbf{N}$ et al. (2000) The European Sero-Epidemiology Network: standardizing the enzyme immunoassay results for measles, mumps and rubella. Epidemiology and Infection 125, 127-141.
34. Backhouse JL et al. (2007) Population-based seroprevalence of Neisseria meningitidis serogroup $\mathrm{C}$ capsular antibody before the introduction of conjugate vaccine, in Australia. Vaccine 25, 1310-1315.

35. Ceyhan M et al. (2007) Age-specific seroprevalence of serogroup C meningococcal serum bactericidal antibody activity and serogroup A, C, W135 and Y-specific IgG concentrations in the Turkish population during 2005. Vaccine 25, 7233-7237.

36. Findlow $\mathbf{H}$ et al. (2019) Kinetics of maternally-derived serogroup A, C, Y and $\mathrm{W}$-specific meningococcal immunoglobulin $\mathrm{G}$ in Malian women and infants. Vaccine 37, 2477-2481.

37. Hahne S et al. (2009) Rubella outbreak in the Netherlands, 2004-2005: high burden of congenital infection and spread to Canada. Pediatric Infectious Disease Journal 28, 795-800.

38. van den Hof S, Conyn-van Spaendonck MA and van Steenbergen JE (2002) Measles epidemic in the Netherlands, 1999-2000. Journal of Infectious Diseases 186, 1483-1486.

39. Wielders CC et al. (2011) Mumps epidemic in orthodox religious lowvaccination communities in the Netherlands and Canada, 2007 to 2009. EuroSurveillance 16, 14-22.

40. de Greeff SC et al. (2006) Protection from routine vaccination at the age of 14 months with meningococcal serogroup $\mathrm{C}$ conjugate vaccine in the Netherlands. Pediatric Infectious Disease Journal 25, 79-80.

41. Ruijs WL et al. (2011) Religious subgroups influencing vaccination coverage in the Dutch Bible belt: an ecological study. BMC Public Health 11, 102.

42. Moore SE et al. (2006) Effect of month of vaccine administration on antibody responses in The Gambia and Pakistan. Tropical Medicine and International Health 11, 1529-1541.

43. Martins C et al. (2014) Measles virus antibody responses in children randomly assigned to receive standard-titer edmonston-Zagreb measles vaccine at 4.5 and 9 months of age, 9 months of age, or 9 and 18 months of age. Journal of Infectious Diseases 210, 693-700.

44. Linder $\mathbf{N}$ et al. (2011) Effect of season of inoculation on immune response to rubella vaccine in children. Journal of Tropical Pediatrics 57, 299-302.

45. Haralambieva IH et al. (2014) Associations between race, sex and immune response variations to rubella vaccination in two independent cohorts. Vaccine 32, 1946-1953.

46. Wright CY et al. (2017) Sun protection to improve vaccine effectiveness in children in a high ambient ultraviolet radiation and rural environment: an intervention study. BMC Public Health 17, 37.

47. Amanna IJ and Slifka MK (2010) Mechanisms that determine plasma cell lifespan and the duration of humoral immunity. Immunological Reviews 236, $125-138$

48. Waaijenborg S et al. (2013) Waning of maternal antibodies against measles, mumps, rubella, and varicella in communities with contrasting vaccination coverage. Journal of Infectious Diseases 208, 10-16.

49. Brinkman ID et al. (2019) Early measles vaccination during an outbreak in the Netherlands: short-term and long-term decreases in antibody responses among children vaccinated before 12 months of age. Journal of Infectious Diseases 220, 594-602.

50. Benn CS et al. (2013) A small jab - a big effect: nonspecific immunomodulation by vaccines. Trends in Immunology 34, 431-439.

51. Knol M et al. (2013) Large ongoing measles outbreak in a religious community in the Netherlands since May 2013. EuroSurveillance 18, pii=20580.

52. van Twillert I et al. (2014) Age related differences in dynamics of specific memory B cell populations after clinical pertussis infection. PLoS ONE 9, e85227.

53. Gouma S et al. (2016) Mumps-specific cross-neutralization by MMR vaccine-induced antibodies predicts protection against mumps virus infection. Vaccine 34, 4166-4171.

54. Sallusto F, et al. (2010) From vaccines to memory and back. Immunity 33, 451-463. 